\title{
Proficient Mining of Informative Gene from Microarray Gene Expression Dataset Using Machine Intelligence
}

\author{
Priya Ravindran ${ }^{\mathrm{a}, 1}$, S. Jayanthi ${ }^{\mathrm{a}}$, Arun Kumar Sivaraman ${ }^{\mathrm{b}}$, Dhanalakshmi R ${ }^{\mathrm{c}}$, \\ A.Muralidhar ${ }^{\mathrm{b}}$, Rajiv Vincent ${ }^{\mathrm{b}}$ \\ a Dept of CSE, Agni College of Technology, Chennai, India \\ ${ }^{b}$ School of Computer Science and Engineering, VIT University, Chennai, India \\ ${ }^{c}$ Department of CSE, KCG College of Technology, Chennai, India
}

\begin{abstract}
The quick improvement of DNA microarray innovation empowers analysts to quantify the expression levels of thousands of genomic data and permits scientists effortlessly pick up and understanding the mind-boggling prediction in tumors based on genomic expression levels. The application in malignancy has been demonstrated and extraordinary achievement has been performed in both conclusion and clarification using the neurotic methodologies. In many cases, DNA microarray information about gene contains a large number of qualities and the majority of them are turned out to be uninformative and excess. In the interim, little size of tests of microarray information undermines the determination precision of factual models. In this way, choosing profoundly discriminative qualities from crude quality genetic expression can enhance the execution of genetic prediction and chopped down the cost of medicinal analysis. Pearson Correlation based Feature Selection strategy with machine learning methodologies is effective to locate a conspicuous arrangement of components which can be utilized to anticipate and idealize the blend of quality to analyze the disease. As conflicting to the customary cross approval, filter one cross approval technique is connected for the analyses. As needs be, the proposed blend between the PCBFS and Machine Learning methodology is an effective apparatus for disease grouping and can be actualized as a genuine clinical supportive system.
\end{abstract}

Keywords.microarray gene dataset, machine intelligence, human cancer classification, feature engineering.

\section{Introduction}

Precise disease determination is essential for the effective utilization of particular treatments. In spite of the fact that tumor order has enhanced throughout the most recent decade, there is as yet a requirement for a completely robotized and less subjective technique for malignancy conclusion. Late reviews showed that DNA microarrays could give valuable data to malignancy characterization at the quality expression level because of their capacity to gauge the plenitude of delivery person ribonucleic corrosive transcripts for a huge number of qualities at the same time.

A small number of machine-learning computations have as of now been associated for tumors classification by utilizing microarray information. By utilizing the Voting

${ }^{1}$ Priya Ravindran, Dept. of Computer Science and Engineering, Agni College of Technology, Chennai, India 
maps to break down intense lung (Golub et al., 1999). Bolster trajectory machines are connected to multiclass growth determination by (Ramaswamy et al., 2001). Various leveled bunching is utilized to examine colon cancer (Alon et al., 1999). The superlative characterization outcomes are accounted for by (Li et al., 2003) and (Antonov et al., 2004). (Li et al., 2006) utilized a lead revelation strategy along with greatest edge straight system. In this research, we deliberate two traditional ways to deal with highlight subclass choice, by particularly considering, wrapper and channel tactics, for quality determination. Wrappers and channels contrast by the way they assess include subsets. Channel approaches evacuate immaterial components as per general qualities of raw information. Wrapper methods, by complexity, smear machine mastering calculations to highlight datasets and utilize cross-approval to assess the score of highlight subsets. Most techniques for quality determination for microarray information investigation concentrate on channel approaches, in spite of the fact that there are a couple of distributions on smearing wrapper methods (Inza et al., 2004). This research paper is technically organized as follows. We start with a short prologue to highlight subclass choice, trailed by a portrayal of highlight wrappers, channels and FSC, which is basically a channel calculation. We talk about the points of interest and impediments of utilizing wrappers and channels to choose featured subclasses. From there on, we display the test comes about on intense lung and lymphoma dataset information. The preceding segment talks about the outcomes and finalize this research paper.

\section{Existing Methods}

Given a microarray growth informational collection $\mathrm{D}$, which comprises $\mathrm{n}$ tests from various malignancy sorts or subclasses, we need to assemble a scientific display which will delineate examples to their subclasses. Each specimen has $\mathrm{m}$ qualities as its elements. The supposition here is that not all qualities restrained by a microarray dataset are identified with tumor arrangement. A few qualities are superfluous and excess from the machine learning perspective. It is notable that the incorporation of unimportant and repetitive in-arrangement may hurt execution of some machine learning calculations.

\section{$2.1 \quad$ Distance Measures}

The partition estimations compute the imminence of the things in perspective on different characteristics. These estimations are disengaged into two social occasions as comparability or uniqueness estimations. Likeness estimations measure how relative any match of individuals is and all around take esteems in the region of 0 and 1 . The regard 0 means "no likeness" while 1 suggests an "all out closeness" (Tan, 2006). On the other hand, distinction estimations measure how far the articles are. Subsequently, the disparity estimations can be taken as the detachment between sets. Articles with divergence score close to 0 are believed to be nearby. The similarity among the things gets worse as the divergence score lengthens. The multiple sorts of divisions make the divergences among the things increases gradually as the partition among the items increases. Minimum highlight is agreed to the greater partitions. The three estimations obtained from the mentioned situations, describes the divergences among the objects in the sense of their incomparable complexities. In course of action, along these lines, 
these estimations will evaluate the degree differentiates among the recognitions at core interests.

An additional measurement is notorious as the cosine-edge evacuate. It basically evaluates the cosine regard between the different vectors. The position among two vectors became increasing and the measurement will be progressively similar to 1 , exhibits that two vectors became similar to one another. Along these lines, it varies well by appointing a similarity metric.

$$
\text { Cosine-Angle Distance: } d(x, y)=1-\frac{\sum_{i=1}^{n} x_{i} y_{i}}{\sqrt{\sum_{1}^{n} x_{i}^{2} \sqrt{\sum_{1}^{n} y_{i}^{2}}}}
$$

Another measurement was projected by (Möller-Levet et al., 2005) and termed as Petite Time sequence Space. It was described to check the contour mismatches amid the short-range course of action. This measurement, on a very basic level, measures the partition between the inclinations of the time-course of action at each time break, and usages their sums over different time centers. Petite Time sequence Space partition can be estimated as pursues:

$$
\text { Petite Time sequence Space: } d(x, y)=\sum_{i=1}^{n-1}\left(\frac{x_{i+1}-x_{i}}{t_{i+1}-t_{i}}-\frac{y_{i+1}-y_{i}}{t_{i+1}-t_{i}}\right)
$$

Where $\mathrm{x}$ and $\mathrm{y}$ are the time intervals with $\mathrm{n}$ time centers; and $\mathrm{t}_{\mathrm{i}}$ exhibits the consequent time of the $\mathrm{i}^{\text {th }}$ recognition. Rather than the underlying three estimations portrayed in this section, the estimations gained from above condition get the dissimilarities between the things in perspective on their shapes. All things considered, every partition metric describes the uniqueness between the things by using one of the characteristics which are, all around, the size and grade contrasts.

\section{Tailored Analytic Hierarchy Process}

Using pairwise comparisons, the relative importance of one gene over other can be expressed. Here we show how to get a ranking of properties from a pairwise matrix. Mathematically saying, Eigen Vector is the best approach. To achieve this ranking, a quick computational way is to lift the pairwise matrix to powers that are squared each time successively. Then the sums of the rows are measured and normalized. If the difference between these quantities is smaller than the specified value in two consecutive measurements, the machine is instructed to stop. The gene similarity scores are represented in the matrix below: Using pairwise comparisons, the relative importance of one gene over other can be expressed. Here we show how to get a ranking of properties from a pairwise matrix. Mathematically saying, Eigen Vector is 
the best approach. To achieve this ranking, a quick computational way is to lift the pairwise matrix to powers that are squared each time successively. Then the sums of the rows are measured and normalized. If the difference between these quantities is smaller than the specified value in two consecutive measurements, the machine is instructed to stop.

Eigen Value can be calculated by,

$$
\begin{aligned}
& \lambda_{1}=\left(\left[\operatorname{SM}_{(1,1)}, \operatorname{SM}_{(1,2)} \ldots \ldots, \mathrm{SM}_{(1, \mathrm{n})}\right] \cdot\left[\epsilon_{1}, \epsilon_{2}, \ldots ., \epsilon_{\mathrm{n}}\right]^{\mathrm{T}}\right) / \epsilon_{1} \\
& \lambda_{2}=\left(\left[\mathrm{SM}_{(2,1)}, \mathrm{SM}_{(2,2)} \ldots \ldots, \mathrm{SM}_{(2, \mathrm{n})}\right] \cdot\left[\epsilon_{1}, \epsilon_{2}, \ldots ., \epsilon_{\mathrm{n}}\right]^{\mathrm{T}}\right) / \epsilon_{2} \\
& \lambda_{3}=\left(\left[\mathrm{SM}_{(3,1)}, \mathrm{SM}_{(3,2)} \ldots \ldots ., \mathrm{SM}_{(3, \mathrm{n})}\right] \cdot\left[\epsilon_{1}, \epsilon_{2}, \ldots ., \epsilon_{\mathrm{n}}\right]^{\mathrm{T}}\right) / \epsilon_{3} \\
& \lambda_{\mathrm{n}}=\left(\left[\mathrm{SM}_{(\mathrm{n}, 1)}, \mathrm{SM}_{(\mathrm{n}, 2)} \ldots \ldots, \mathrm{SM}_{(\mathrm{n}, \mathrm{n})}\right] \cdot\left[\epsilon_{1}, \epsilon_{2}, \ldots ., \epsilon_{\mathrm{n}}\right]^{\mathrm{T}}\right) / \epsilon_{\mathrm{n}}
\end{aligned}
$$

\section{Results and Discussion}

We used microarray analysis as a case study, where genes with identical expressions or similar molecular functions were grouped together, to assess the efficacy of the proposed formulation. In particular, on three benchmark microarray gene expression datasets, the proposed feature selection method is tested and evidence is given that the proposed method provides more precise results than the state-of-the-art methods of gene selection. The results are discussed based on three different cancer datasets taken in various time intervals. Overlap matrices are used to evaluate the performance of different data mining approaches.

\subsection{Overlap Matrix in Gene Selection Approaches: For TCL microarray}

The overlap between the first 30 nominated human genes in the six gene assortment approaches for the TCL dataset is shown in table 1 . There are 28 common genes between Tailored-AHP and Wilcoxon out of 30 selected genes, and also 27 common genes between Tailored-AHP and ROC out of 30 selected genes.

Table 1. overlap matrix between gene assortment techniques: for TCL dataset

\begin{tabular}{ccccccc}
\hline & T-test & Entropy & ROC & Wilcoxon & SNR & TAHP \\
\hline T-test & 30 & 18 & 26 & 28 & 14 & 26 \\
Entropy & 18 & 30 & 17 & 18 & 9 & 15 \\
ROC & 26 & 17 & 30 & 28 & 18 & 27 \\
Wilcoxon & 28 & 18 & 28 & 30 & 16 & 28 \\
SNR & 14 & 9 & 18 & 16 & 30 & 19 \\
TAHP & 26 & 15 & 27 & 28 & 19 & 30 \\
\hline
\end{tabular}


The normalized LOOCV accurateness among multiple gene datasets of the four clustering techniques, i.e. K-Means, Hierarchical, Apriori, and DSSOM is evaluated in table 2 for the TCL dataset.

Table 2. LOOCV precision on TCL dataset

\begin{tabular}{ccccccc}
\hline & & Entropy & ROC & Wilcoxon & SNR & TAHP \\
\hline K-Means & 93.67 & 96.30 & 93.82 & 97.60 & 95.23 & 96.43 \\
Apriori & 94.63 & 92.46 & 93.74 & 93.19 & 92.18 & 94.68 \\
Hierarchical & 97.49 & 93.38 & 96.84 & 97.34 & 97.57 & 95.74 \\
DSSOM & 98.32 & 97.56 & 94.38 & 96.37 & 98.23 & 99.21 \\
\hline
\end{tabular}

\section{Summary and Conclusion}

In order to evaluate and interpret highly significant data (such as microarray datasets), several approaches have been explored that combine feature selection and classification, most of which are considered to be obsolete and insignificant. Until running either classification techniques on the selected features, or running a further 'combined' feature selection/classification process, it has generally been found that previous feature selection is advantageous. We proposed a new mechanism to take into account the ranking results of individual gene selection methods including t-test, entropy, receiver operating characteristic curve and signal to noise ratio for informative gene selection by using Statistical Analysis with Pearson Correlation System and performing modifications in the traditional Analytic Hierarchy Process (named as Tailored-AHP). For the reason that the grading would be diverse for each technique, the grading result of a solitary technique is forever uncertain. In conjunction with the Pearson Correlation Tool, the Tailored-AHP shows heftiness and ascendancy assessed for different gene ranking approaches. Gene ranking approaches results are evaluated by leaving one out cross validation (LOOCV). The use of Tailored-AHP on average produces approximately 99 percent LOOCV accuracy in the TCL dataset, which is the greatest statistic compared to those of the remaining approaches. Similarly, in the lung dataset, the highest LOOCV precision, more than 98 percent, is also the product of the Tailored-AHP with Pearson Correlation method. The Tailored-AHP also contributes to the greatest LOOCV precision at more than 96 percent in the breast dataset.

\section{References}

[1] Agathanggelou, A., Bieche, I., Ahmed-Choudhury, J., Nicke, B., Dammann, R., Baksh, S., Gao, B., Minna, J., Downward, J., Maher, E., Latif, F. Identification of novel gene expression targets for the ras association domain family 1 (rassfla) tumor suppressor gene in nonsmall cell lung cancer and neuroblastoma. Cancer Res, 2003, 63 (17), 5344-5351.

[2] Alizadeh, A.A., M.B. Eisen, R.D., Ma, C., Lossos, I., Rosenwald, A., Boldrick, J., Sabet, H., Tran, T., Yu, X., Powell, J., Yang, L., Marti, G., Moore, T., Hudson, J.J., Lu, L., Lewis, D., Tibshirani, R., Sherlock G., Chan, W., Greiner, T., Weisenburger, D., Armitage, J., Warnke, R., Levy, R.,Wilson,W., Grever, M., Byrd, J., Botstein, D., Brown, P., Staudt, L.M.. Distinct types of diffuse large b-cell lymphoma identified by gene expression profiling. Nature, 2000, 403 (6769), 503-511. 
[3] Alon, U., Barkai, N., Notterman, D., Gish, K., Ybarra, S., Mack, D., Levine, A. Broad patterns of gene expression revealed by clustering analysis of tumor and normal colon tissues probed by oligonucleotide arrays. Proc. Natl. Acad. Sci., 1999, 96 (12), 6745-6750.

[4] Antoniadis, A., Lambert-Lacroix, S., Leblanc, F. Effective dimension reduction methods for tumor classification using gene expression data. Bioinformatics, 2003, 19, 563-570.

[5] Antonov, A.V., Tetko, I.V., Mader, M.T., Budczies, J., Mewes, H.W. Optimization models for cancer classification: extracting gene interaction information from microarray expression data. Bioinformatics, 2004, 20, 644-652.

[6] Crawford, A., Beckerle, M. Purification and characterization of zyxin, an 82,000-dalton component of adherens junctions. J. Biol. Chem, 1991, 266 (9), 5847-5853.

[7] Fayyad, U., Irani, K. Multi-interval discretization of continuousvalued attributes for classification learning. Proceedings of IJCAI-93, 13th International Joint Conference on Artificial Intelligence,1993, pp. 1022-1027.

[8] Frank, E., Hall, M., Trigg, L., Holmes, G., Witten, I.H. Data mining in bioinformatics using Weka. Bioinformatics, 2004 20, 2479-2481.

[9] Ramakrishan.M et.al.,Footprint Based Recognition System. in the month of April for the International Journal Communication in Computer and Information System (CCIS) Journal (Springer) Volume 147, Part 3, 358-367, DOI: 10.1007/978-3-642-20573-6_63, April 2011

[10] M. Indhumathi et.al , "Healthcare Management of Major Cardiovascular Disease-A review", 2021 6th International Conference on Inventive Computation Technologies (ICICT), (DOI: 10.1109/ICICT50816.2021.9358519)

[11] Ambeth Kumar.V.D, Dr.S.Malathi, V.D.Ashok Kumar (2015) .Performance Improvement Using an Automation System for Segmentation of Multiple Parametric Features Based on Human Footprint. for the Journal of Electrical Engineering \& Technology (JEET), vol. 10, no. 4, pp.1815-1821, 2015. [http://dx.doi.org/10.5370/JEET.2015.10.4.1815]

[12] Ambeth Kumar.V.D et,al, .Enhancement in Footprint Image using Diverse Filtering Technique.

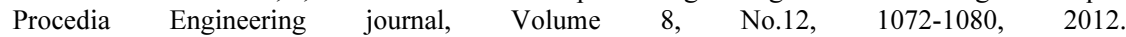
[doi:10.1016/j.proeng.2012.01.965] 